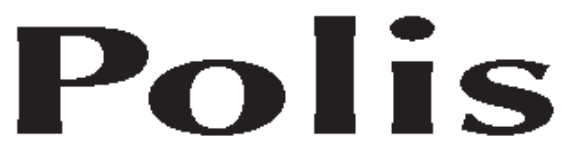

\title{
Humanismo radical, decrecimiento y energía: una lectura de las ideas de Iván Illich
}

\author{
Roberto Espejo*
}

\begin{abstract}
"Por supuesto, aquellos que preconizan un "cambio radical de la estructura política y social" pasan por ser utopistas incorregibles, mientras que aquellos que no son capaces de ver más allá de su nariz en dos años en el futuro, son evidentemente los realistas" (Cornelius Castoriadis, citado por Ridoux, p.91).
\end{abstract}

Resumen: En este artículo presentamos una descripción de la crítica de Iván Illich a la sociedad industrial, específicamente en relación al problema de la energía y de la equidad. Especial atención es dada al concepto de convivencialidad y a sus posibles repercusiones para enfrentar los problemas energéticos. Una discusión en torno a la manera de Illich de criticar a la sociedad es presentada, en relación a lo que ha sido llamado un "humanismo crítico" y al problema de las necesidades individuales.

Palabras claves: Illich, energía, convivencialidad, humanismo radical, decrecimiento.

\section{Radical humanism, decreasement and energy: a reading of the ideas of Iván Illich}

Abstract: In this article we present Iván Illich's critique to industrial society, specifically in relation to the problem of energy and equity. Special attention is given to the concept of conviviality and its possible offshoots to face energetic problems. A discussion about Illich's way of critiquing society is presented, in relation to what has been called a "radical humanism" and to individual needs.

Key words: Illich, energy, conviviality, radical humanism, decreasement.

Recibido: 18.09 .08

Aceptado: 24.11.08

*Universidad de Paris 8, Paris, Francia. Email: roberto.espejo@gmail.com 


\section{Introducción}

Hay situaciones que son evidentes por sí mismas. Hay otras que pueden parecer evidentes pero que esconden errores de razonamiento, los cuales -dependiendo de la situación- pueden estar muy o poco escondidos. Otras situaciones se presentan como completamente auto-evidentes y a veces quedan en una especie de espacio neutro, donde nadie se hace cargo.

El tema de la energía y de los recursos es una de estas situaciones. Vivimos en un planeta y utilizamos sus recursos, pero lo hacemos como si dicho planeta tuviera fuentes ilimitadas de éstos. ¿Por qué nos comportamos de esta manera? Hemos construido un sistema económico basado en el mercado y en el desarrollo. Pero ¿qué entendemos por tal? La producción de bienes y servicios, el movimiento creativo y empresarial nos transforman en productores de riqueza. Nada de esto es intrínsecamente malo, al contrario, creo que la labor de transformación del mundo es algo deseable, un movimiento donde los seres humanos tienen la posibilidad de crear estructuras, de intentar formas de organización, de equivocarse y de tener aciertos. La pregunta está en el cómo llevamos a cabo dicha actividad en el mundo. Podríamos decir que lo importante es que dicha actividad sea llevada a cabo de manera ética.

Por esto, y para no complicarnos en discusiones filosóficas, entenderemos simplemente que dicha actividad debiera desarrollarse de manera tal que la vida de un grupo de personas no sea perjudicada por aumentar la calidad de vida de otros. Pero dicho criterio puede ser visto en un momento de tiempo o bien de forma transversal. Es por esto que el problema de los recursos y de la energía es doble: por una parte su distribución (desigualdad) y por otra su permanencia en el tiempo (sustentabilidad).

Pero, ¿en qué consiste este desarrollo? Todo parece indicar que se trataría de la producción incesante de bienes y servicios, siempre pensando en satisfacer un conjunto de necesidades de la población, con el consecuente consumo de energía. Para este fin, hemos creado instituciones que regulan dicho proceso. Pero ¿cuál es la naturaleza de estas necesidades que buscan ser satisfechas? ¿Podemos poner un fin a las necesidades, satisfaciéndolas? Es cierto que lo que hoy es considerado como esencial para vivir en sociedad, hace 50 años no lo era. Más aún, los avances de la tecnología nos ponen en la situación de deber actualizarnos día a día. Hace 10 años era pensable vivir sin un teléfono celular, hoy se hace difícil.

No obstante, este desarrollo sigue estando basado en un supuesto que choca con nuestro sentido común. Si cada país busca el desarrollo económico y esto implica la utilización de recursos, y además conocemos las proyecciones demográficas para los años a venir, ¿qué pasará con las fuentes de dichos recursos? No se trata de un tema ideológico sino que práctico. ¿Tenemos razones para creer que tendremos siempre recursos ilimitados 
o se trata más bien de un cerrar de ojos irracional? A veces la situación da la impresión de constituirse en una creencia ciega, de que las cosas se solucionarán por arte de magia o bien

que "la ciencia" - que en nuestros días ha tomado el rol de un cuerpo eclesiástico dotado de infalibilidad casi divina - encontrará las vías necesarias para solucionar nuestros problemas energéticos ${ }^{1}$. Una crítica de esta situación va más allá de un análisis basado en un marco teórico o político definido y se inserta en lo que podríamos llamar una constatación de sentido común. Un símil cuya fuente no he podido rescatar es muy gráfico: es como si un chofer de automóvil fuera a chocar contra una muralla y al verla acelerara aún más. Nuestro chofer ve la muralla, pero cree que se liberará de ella aumentando la velocidad. Quizás en su espíritu reine el wishful thinking y crea que podrá atravesarla.

Estos tipos de cuestionamientos fueron desarrollados por Ivan Illich (1926-2002) en una serie de escritos que fueron bastante debatidos entre los años 70 y 90 . Illich se volvió famoso en los años 70 por su crítica al sistema escolar, con su libro "La Sociedad Desescolarizada" y varios otros escritos y conferencias, que cubría sólo una pequeña parte de una crítica a la sociedad industrial. Dentro de esta crítica, el problema de la energía está presente y debiéramos decir que cumple un papel fundamental en su forma de analizar la situación de nuestra civilización.

En este artículo quisiéramos exponer algunas de sus ideas principales para resucitar el debate en torno a su obra. Sus escritos cubren muchos dominios de la sociedad -siempre como parte de este análisis- pero ciertamente pueden describirse utilizando algunos denominadores comunes. La manera de Illich de mirar el mundo consiste en una forma específica de crítica, en el sentido que usualmente se le da a este término en los círculos académicos. $^{2}$ Es por esto y por lo radical de sus ideas, que nos parece pertinente discutirlas.

\section{Ivan Illich}

Illich nació en 1926 en Viena, Austria. Era hijo de un padre croata católico y de una madre judía. Huyendo del antisemitismo de la época, emigraron a Italia donde hizo estudios de cristalografía en la Universidad de Florencia, obteniendo un diploma en esa materia. En

\footnotetext{
${ }^{1}$ Por ejemplo, el sacar energía del vació o la fusión en frío. Ideas que pueden transformar efectivamente nuestra civilización, pero que hasta que no se logren implementar no pasan de ser un relato de ciencia ficción. Además, alguien podría argumentar, muchas opciones existen (energía solar, eólica, etc.) pero no son masificadas por razones de poder y de control social. ¿Son nuestros ministerios de energía y grandes corporaciones aparatos ideológicos althusserianos de un estado transnacional y mundialmente organizado? Conspiraciones podemos ver en muchas partes, pero lo que es innegable es que nuestro actuar no es el más racional de todos.

${ }^{2}$ Crítica cercana a la realizada por Jacques Ellul en Francia y por Paul Goodman en Estados Unidos.
} 
1943 buscando transformarse en sacerdote estudia teología y filosofía en la Universidad Gregoriana del Vaticano. Posteriormente en 1951 se ordena, al mismo tiempo que obtiene

un doctorado en historia en la Universidad de Salzburgo. No queriendo desarrollar una carrera eclesiástica en el seno de la iglesia, Illich parte a Nueva York (buscando trabajar con unos manuscritos de San Alberto Magno) donde se transformará en párroco de una iglesia puertorriqueña entre 1951 y 1956. En 1956, con treinta años, asume como vicerrector de la Universidad Católica de Puerto Rico, la cual deja en 1960 para establecerse en Cuernavaca, México.

En 1961 funda en esta ciudad el Centro Intercultural de Información (CIDOC), el cual fue considerado por muchos como una "universidad informal" donde se estudiaba el español, pero sobre todo se trabajaba sobre los problemas del desarrollo. De hecho, el centro estaba pensado como un espacio para capacitar a misioneros latinoamericanos en su trabajo en América Latina. Pronto el centro demostró hacer más que eso, teniendo una gran expansión teórica y siendo conocido internacionalmente. Como señala Marcela Gajardo: "Desde el año de su creación hasta mediados de los 70, el CIDOC fue un lugar de encuentro para muchos intelectuales americanos y latinoamericanos dedicados a la reflexión sobre la educación y la cultura. Allí se impartían cursos de español y se organizaban talleres sobre temas sociales y políticos" (Gajardo, 1993). A raíz de la tendencia del CIDOC, Illich tuvo problemas con el Vaticano, lo cual lo llevó en 1969 a abandonar su función como sacerdote y a transformarse en laico.

En los años 70 Illich publicó las obras que lo harán conocido como crítico de la sociedad industrial y de sus instituciones: Celebration of Awareness en 1970, Deschooling Society (La Sociedad desescolarizada) en 1971, Tools for Conviviality (La convivencialidad) en 1973, Energy and Equity (Energía y Equidad) en 1973 y Medical Nemesis (Némesis médica) en 1975. Como lo señalan Jean Robert y Valentina Borremans en el prefacio de las Obras Completas de Illich -libro aparecido en 2003 y editado por Fayard- Illich se refería a estos cuatro libros como sus «panfletos» y ellos presentaban un conjunto coherente con un propósito común. (Illich 2003: 12).

Como ya comentamos anteriormente, quizás una de las áreas donde Illich es más conocido sea como crítico del sistema escolar, ámbito donde sus planteamientos por eliminar la escuela obligatoria fueron muy debatidos en los años 70 y cuyas repercusiones duran hasta hoy en día, aunque moduladas por el tiempo. Particularmente interesante es la discusión entre Illich y Paulo Freire, en torno al problema de la concientización y al rol de la escuela. ${ }^{3}$ A pesar de ser este uno de los temas que se relaciona por lo general más con Illich, se trata de un tema que está englobado en una posición crítica de la sociedad bastante más amplia.

\footnotetext{
${ }^{3}$ Ver el trabajo de Gustavo Esteva, Dana Stuchul y G. Prakash citado en la bibliografía.
} 
y crítico publicando otros libros y artículos. En los años 90 se le podía ver un tiempo en su casa en Cuernavaca (la ex sede del CIDOC), donde rechazaba casi toda entrevista, en Alemania (Bremen) donde trabajaba con un grupo de estudiantes y amigos temas relacionados con la historia antigua y con la crítica de la sociedad industrial y en la Universidad de Pensilvania, donde daba también conferencias y estaba encargado de un seminario sobre la fillosofía de la tecnología. Illich murió en Bremen, Alemania, en 2002.

\section{La crítica a la sociedad moderna}

\section{Una crítica diferente}

Es interesante notar que cuando hablamos de crítica social siempre somos llevados a un imaginario que contiene los rostros y las invocaciones teóricas de la Escuela de Francfort. Muchas obras han sido escritas desde los años 70 narrando la historia de esta escuela (Wiggerhaus, 1993; Jay, 1977, Assoun, 2001, Vincent, 1976). Dicho movimiento se constituyó en los años 30 en Alemania como un Instituto de investigación social que tenía una línea de discurso explícitamente neo-marxista. Figuras como Horkheimer, Adorno, Fromm, Marcuse (y posteriormente Habermas) fueron los pilares de este movimiento. Como señala Kellner: "el rol histórico llevado a cabo por el Instituto de Investigación Social fue preservar la teoría marxista de una degeneración ortodoxa cerrada, de desarrollar dicha teoría aplicándola a problemas sociales e históricos diferentes" (Kellner 1975: 142).

La producción teórica de estos personajes tomó en el tiempo direcciones distintas, pero siempre consistió en un análisis de la sociedad y en la aplicación de la denominada teoría crítica a los múltiples aspectos de ésta. En la década de los 60 personajes como Marcuse $^{4}$ se transformaron en los referentes de la revuelta estudiantil (el Mayo 68 Francés y las manifestaciones en la Universidad de Berkeley en Estados Unidos). Por otra parte, Erich Fromm desarrolló más el lado existencial y religioso de esta mirada de la sociedad, separándose del tipo de textos producidos por lo que ha sido llamada la Escuela de Francfort de la primera generación.

De manera más o menos paralela, Ivan Illich realizó una crítica de la sociedad alejada del cuadro freudo-neo-marxista desarrollado por la Escuela de Francfort. Se trata de una

\footnotetext{
${ }^{4}$ Con sus conocidos textos "El Hombre Unidimensional” y "Eros y Civilización”. Posteriormente Marcuse desarrolló sus argumentos en torno a la aplicación de la Estética en este análisis social. Ver por ejemplo "La dimensión Estética” y "Hacia la Liberación: más allá del hombre unidimensional".
} 
crítica no marxista y que mantiene la exposición de sus ideas en un terreno mucho menos teórico -en el sentido del discurso académico habitual- que el caso de la crítica à la

Francfort. La crítica de Illich busca ${ }^{5}$ no ser ideológica, sino que práctica, hasta cierto punto basada en el sentido común. Creemos que para él el problema estaba en hacer frente a una realidad y constatarla, no buscando construir políticas en base a supuestos predeterminados. Erich Fromm, en el prefacio escrito para el libro de Illich "Celebration of Awarness" -que podríamos traducir como "la celebración de la conciencia" o del "darse cuenta"- define el tipo de crítica Illichiana como un "radicalismo humanista": "Antes de toda reflexión, el radicalismo es llevado a adoptar como lema 'de omnibus dubitandum'; hay que dudar de todo, en particular de aquellos conceptos ideológicos que están bien anclados en la opinión pública y que se transforman en axiomas" (Erich Fromm, en Illich, 2003: 41).

\section{Los umbrales de desarrollo}

En este contexto Illich propuso su teoría de los "umbrales de desarrollo". La idea es simple: en muchos ámbitos (notoriamente en el caso de la educación, del transporte y de la salud) el desarrollo de instituciones para administrar y promover los mecanismos para satisfacer las necesidades de dichos dominios comienza a actuar en contra el objetivo de la institución. Todo esto a partir de un punto de inflexión, donde la eficiencia del mecanismo propuesto empieza a ser decreciente. De esta manera, el sistema de educación empieza a impedir que la gente se eduque, el sistema de salud enferma a la gente y el sistema de transporte -considerando todos sus costos- se transforma en un sistema muy poco eficaz. Podemos ilustrar la idea pensando en el tráfico: hasta un cierto punto el uso de un automóvil es práctico, debido a su velocidad. Pero si el sistema de los automóviles crece, y se producen estancamientos, la velocidad efectiva de este dispositivo corre riesgos de disminuir. Existiría entonces un límite al crecimiento del sistema, a partir del cual el sistema en sí se vuelve ineficaz.

El problema se encuentra en la consideración de los límites a nuestras acciones como civilización. ¿Podemos pensar en hacer crecer nuestra producción y obtener satisfacción a nuestras necesidades siempre de manera ilimitada? Sin entrar aún al tema de los productos, de la necesidad y de la finitud, se trata de considerar el crecimiento industrial desde una perspectiva lógica. Una sociedad debe "reconocer los límites a la manipulación pedagógica y terapéutica que puede ser exigida por el crecimiento industrial y que nos obliga a mantener ésta sin sobrepasar ciertos umbrales críticos" (Illich 2003a: 454). La existencia de un punto a partir del cual el crecimiento de un sistema montado para realizar una tarea se vuelve ineficiente es la idea de base.

\footnotetext{
${ }^{5}$ Que lo logre o no, ese es otro problema.
} 
REVISTA POLIS

VOL. $7 \mathrm{~N}^{\circ} 21,2008$

PRÒLOGO, págs., 63-79

( ) EDITORIAL DE LA UNIVERSIDAD BOLIVARIANA DE CHILE, 2008

Se trata finalmente de incluir el problema de la escala humana en una dinámica de crecimiento exacerbado. Esto es cierto también para el uso de la energía, como lo señala Illich en su libro "Energía y Equidad". Detrás de esta discusión se esconde ciertamente la

noción -de la cual por lo general no estamos conscientes- de la finitud de la vida humana, siempre versus una lógica de crecimiento infinito. ¿No es acaso cierto que individualmente no tenemos consciencia de nuestra propia finitud? ¿No es cierto que vivimos como pensando en que siempre estaremos aquí? Si extrapolamos esta tendencia individual a ver las cosas y a actuar en el mundo, podemos hacernos una imagen de las crisis que podríamos tener que enfrentar de no considerar este tema. Como señala Bernard Delobelle (Delobelle 2008: 50) en relación al crecimiento, "la finitud convoca lo real" y, por lo tanto, el asumir esta finitud puede ser una fuente de sabiduría. ¿Es posible pensar en un proceso social que de cuenta de esta conciencia?

En su texto "Needs" (necesidades) Illich desarrolla el problema de cómo el "desarrollo" se liga con las necesidades que consideramos vitales para el hombre. ¿Fue esto siempre así o hemos construido categorías en base a una miopía histórica? Illich presenta la aparición de estas necesidades como el origen de una categoría antropológica: el homo miserabilis, el hombre que carece de aquello que es considerado por la sociedad como lo básico. Illich señala: "La transformación ocurrió en un par de siglos. Durante este tiempo la certeza de raíz era la evolución, ahora llamada progreso, ahora llamada desarrollo, ahora llamada crecimiento. En este proceso secular los hombres clamaron descubrir "recursos" en la cultura y en la naturaleza -en lo que habían sido cosas comunes para ellos- y los transformaron en valores. El historiador de la escasez relata la historia. Como crema batida que súbitamente se endurece para hacerse mantequilla, el homo miserabilis súbitamente apareció, casi del día a la mañana, como una mutación del homo economicus, el protagonista de la escasez. La generación post segunda guerra mundial observó este cambio en la naturaleza humana desde un hombre común a un hombre necesitado. La mitad de los individuos nacidos en la tierra son de este nuevo tipo" (Illich, 1990).

El progreso y el desarrollo se vuelven términos que es necesario analizar con cuidado. Como señala Gustavo Esteva (en su tiempo amigo y colaborador de Illich) en una entrevista, el subdesarrollo fue introducido como término por Harry Truman en 1949. Esto dividió a la humanidad en dos grupos: los desarrollados y los que no lo eran, generando un ideal que se impuso como un destino obligado para todos. En palabras de Esteva: "Se me pegó el subdesarrollo cuando tenía 13 años, cuando el presidente Truman llegó al cargo y acuñó la palabra "subdesarrollo". Yo fui uno de los 2 billones de personas que ese mismo día nos transformamos en subdesarrollados. No lo éramos. Éramos un tipo diferente de personas y súbitamente todos nos transformamos en subdesarrollados. Si usted se vuelve subdesarrollado es una condición muy humillante. Muy poco digna. Usted no puede confiar en sus instintos. Necesita confiar en los expertos que le traerán el desarrollo" (Esteva, 2005). 
La pregunta es ¿cuál es el costo de este ideal? ¿Es factible pensar en un mundo donde todos satisfagan un conjunto siempre creciente de necesidades? Concretamente, ¿tiene la tierra los recursos necesarios para aquello? Quizás el desarrollo de la humanidad

no implique necesariamente un crecimiento económico sin fin. Quizás las soluciones se encuentren en otros espacios de posibilidades. El pensar en el futuro como un escenario con una demanda siempre creciente de energía para hacer girar las ruedas de nuestra civilización no es algo muy coherente. Aquello presupone fuentes de recursos infinitas y peor aún- no deja espacio a pensar que tal vez no las necesitemos. La mirada de Illich es en este último punto una llamada de atención.

\section{Las herramientas y las instituciones}

Dentro de la dinámica del desarrollo, la utilización de herramientas como extensiones de los sentidos del ser humano es esencial. Pero el uso de tales herramientas implica una cierta conciencia que también podemos ligar a la existencia de un umbral de inflexión, como ya hemos comentado anteriormente. Es por esto que en el caso de las herramientas tomadas en un sentido amplio, y por extensión las instituciones- el hombre se encuentra en una trampa tendida por él mismo. "El uso de la herramienta por el hombre se ha transformado en el uso del hombre por la herramienta. Aquí es necesario reconocer el fracaso. Hace cien años que intentamos hacer trabajar la máquina para el hombre y de educar al hombre a servir a la máquina. Nos damos cuenta ahora que la máquina no "funciona" y que el hombre no sabe cumplir sus exigencias, se hace de por vida su servidor. Durante un siglo la humanidad se ha entregado a una experiencia basada sobre la hipótesis siguiente: la herramienta puede reemplazar al esclavo. Pero, es de manifiesto que con tales designios es la herramienta que ha hecho del hombre su esclavo" (Illich 2003a: 469).

La utilización de herramientas, el movimiento de instrumentalización -que Illich relaciona con la instrumentalización de los sacramentos por parte de la iglesia católica ${ }^{6}-$ de las cosas y de los seres, nos hace llegar a una situación donde ésta se devuelve sobre nosotros mismos. Hoy en día nos hemos transformado en herramientas de un "sistema organizado" - por utilizar la expresión de Paul Goodman. Los seres humanos nos hemos transformados en herramientas, y las instituciones y las máquinas se han transformado en nuestros amos.

Illich plantea de esta manera que cuando una institución se vuelve la única posible fuente de un servicio, como por ejemplo en el caso de la educación, de la medicina o del transporte, ésta deviene un "monopolio radical" (Illich 2003 a: 513). No se trata, como en el caso de la definición habitual de un monopolio, de una firma que monopoliza un cierto servicio: es una institución completa la que lo hace. De esta manera, la gente se ve forzada a ir a la escuela si quiere "educarse", de estar en el sistema de servicio médico si quiere "sanarse" y de utilizar los medios de transporte si quiere "desplazarse". El ponerse al margen de estos servicios transforma a la persona en un outsider, y muchas veces es obligado legalmente a utilizarlos.

\footnotetext{
${ }^{6}$ Ver Illich, 2004, p. 9.
} 


\section{La sociedad convivencial}

Como un contrapeso a esta tendencia Illich propone la noción de pensar una sociedad calificada por lo que él llama "convivencialidad", de reestablecer los lazos entre los seres humanos y de limitar el crecimiento ilimitado más allá de los umbrales naturales. "Yo comprendo la convivencialidad como lo opuesto a la productividad industrial. Cada uno de nosotros se define por su relación al otro y a su entorno y por la estructura profunda de las herramientas que utiliza" (Illich 2003: 470). ¿Cuál es entonces el problema de no tener como centro de la construcción de la sociedad una relación convivencial entre los seres humanos? Vemos marcadamente como Illich propone puertas, quizás líneas de fuga -por utilizar un término deleuziano- a nuestra civilización post-industrial.

¿Pero cómo definir la convivencialidad en relación a la actitud que toma cada ser humano en este escenario? Illich conecta la convivencialidad con la libertad: "La convivenialidad es la libertad individual realizada en la relación de producción, en medio de una sociedad dotada de herramientas eficaces. Cuando una sociedad, no importa cuál, reprime la convivencialidad, más acá de un cierto nivel, se transforma en la presa de la carencia; ya que ninguna hipertrofia de la productividad llegará alguna vez a satisfacer las necesidades creadas y multiplicadas" (Illich 2003a: 471). La convivencialidad implica para Illich una opción en relación a la dualidad tener/ser": "Una sociedad convivencial es una sociedad que da al hombre la posibilidad de ejercer una acción lo más autónoma y lo más creativa, con la ayuda de herramientas menos controlables por otros. La productividad se conjuga en términos de tener, la convivencialidad en términos de ser" (Illich 2003a: 483).

La idea a la base es la construcción de una sociedad en la cual las herramientas dadas para vivir en ella no impidan el ejercicio de la libertad personal. "Esta empresa colectiva limitaría las dimensiones de las herramientas, a fin de defender valores esenciales que yo llamaría: sobrevivencia, equidad, autonomía creadora [...] Estos valores son fundamento para toda estructura convivencial, aun cuando las leyes y la moral varíen de una cultura a otra. Cada uno de estos valores limita, a su manera, la herramienta. La supervivencia es condición necesaria, pero no suficiente, para la equidad: se puede sobrevivir en prisión. La equidad en la distribución de los productos industriales es condición necesaria, pero no suficiente, para un trabajo convivencial: uno puede convertirse en prisionero de la instrumentación. La autonomía, como poder de control sobre la energía, engloba los dos primeros valores citados, y define el trabajo convivencial" (Illich 2003a: 473).

\footnotetext{
${ }^{7}$ Esto nos recuerda a la crítica expuesta por Fromm en su libro "Ser o Tener". Es justo decir que Fromm era íntimo amigo de Illich, y hasta vecino de su casa en Cuernavaca.
} 
Según Illich una herramienta será convivencial en la medida que cada uno pueda utilizarla sin dificultad para los fines que cada uno determine. El uso de esta herramienta no

impide que otro la utilice y nadie debe tener un diploma para hacerlo. Esta "herramienta convivencial" es así un conductor de sentido, un traductor de intencionalidad (Illich 2003b: 485).

La sociedad convivencial se plantea así como una sociedad donde la libertad personal y la autonomía aparecen como elementos articulables con una cierta carencia, impuesta por la definición de desarrollo. Este conjunto de necesidades, como lo señalábamos más arriba, ¿no es la manifestación de un deseo sin límites, como una pulsión básica que tiene un origen en lo profundo de nuestra psiquis, como lo señala Morris Berman (1992)? ¿No es posible ver en esta manifestación una pista para mejor comprender la alienación, tan invocada en los círculos neo marxistas? Las necesidades son ciertamente creadas a través de un aparato social mecánico, en una lógica donde el desarrollo humano es medido -a pesar de los esfuerzos del PNUD de crear índices para cuantificar el "desarrollo humano"por las tasas de crecimiento económico de los distintos países.

Pero recalcamos que la crítica de Illich no es un argumento moral. Se trata para él de constatar que las instituciones, más allá de un cierto umbral, no son eficaces. Luego, él explica esta ineficiencia utilizando su idea de los umbrales de crecimiento. Finalmente él construirá las soluciones posibles utilizando su paradigma de la convivencialidad y de un cambio lógico, que necesitarían las sociedades postindustriales.

\section{Illich y la energía}

El problema del consumo indiscriminado de la energía fue tocado por Illich, pero es interesante que su análisis vaya más allá de un mero argumento ecológico: se trata de una utilización de la energía que "viola a la sociedad y destruye la naturaleza" (Illich 2003b: 383). El argumento de la destrucción de la naturaleza es el usual, tenemos una cantidad de recursos limitada y al parecer no somos conscientes de su finitud. Pero ¿por qué la violación de la sociedad?

En su libro "Energía y Equidad" Illich expresa que detrás del problema energético se encuentra la contradicción inherente de querer lograr "a la vez un estado social fundado sobre la equidad y un nivel siempre más alto de crecimiento industrial" (Illich 2003b: 384). ¿No va esto contra los argumentos del "chorreo" donde los más ricos se hacen más ricos a través del crecimiento y su riqueza "chorrea" hacia los sectores más pobres? Se trata aquí de un problema político. Utilizando su teoría de los umbrales de crecimiento Illich lanza la hipótesis que más allá de un cierto consumo per cápita de energía nos vemos enfrentados a un problema con las libertades individuales. No es tan solo un problema ecológico: "Ciertamente, se comienza a aceptar una limitación ecológica de un máximo de energía 
consumida por persona, viendo en esto una condición para la supervivencia, pero no se reconoce en el mínimo de energía aceptable un fundamento necesario a todo orden social

que sea a la vez justificable científicamente y justo políticamente" (Illich 2003b: 385).

¿En qué radica el problema político de este consumo exacerbado de la energía? Cuando la sociedad se entrega a un consumo desproporcionado de energía per capita, este quantum de energía -como lo llama Illich- "marca el límite donde el orden legal y la organización política deben derrumbarse, donde la estructura técnica de los medios de producción ejerce violencia sobre la estructura social". Cuando este quantum se sobrepasa se substituyen las garantías legales que protegen las iniciativas individuales por una educación que sirve a los objetivos abstractos de una tecnocracia (Illich 2003: 386). Según nuestro autor, al ir más allá de este umbral la energía escapa al poder político y pone al público en manos de argumentos tecnocráticos.

Illich ilustra este juego entre la energía y la equidad analizando el sistema del transporte. Para comenzar, hace la diferencia entre "tránsito" y "transporte": el primer término lo reserva a todo modo de locomoción fundado en la energía metabólica del hombre (como el caminar o la bicicleta) mientras que el segundo recurre a otros tipos de energía. Según él, cuando los seres humanos dependen del "transporte" no solamente para viajes de varios días, sino que para sus trayectos cotidianos, las contradicciones entre la libertad individual y los itinerarios obligados aparecen con toda claridad (Illich 2003b: 393). Volvemos así a encontrar el problema de la "alienación" en nuestra sociedad hiperconsumidora de energía, a pesar de que Illich no utilice a menudo el término: "La industria del transporte da forma a su producto: el usuario. Expulsado del mundo donde las personas son dotadas de autonomía, él también ha perdido la impresión de encontrarse en el centro del mundo. Tiene conciencia de que le falta más y más tiempo, a pesar de que cada día utilice el automóvil, el tren, el autobús, el metro y el ascensor, todo para alcanzar una media de treinta kilómetros, a menudo en un radio de menos de diez kilómetros" (Illich 2003b: 398).

Una crisis energética tiene sentido si pensamos que las necesidades de energía serán siempre crecientes, o hasta constantes en relación a nuestros niveles actuales. Para Illich este término de "crisis" era ambiguo, ya que descansaba sobre la idea -errónea según élque la sociedad necesitaba niveles siempre más elevados de energía. Lo importante para vencer el problema energético era estimar el punto de consumo de energía que sería optimo y luego generar un movimiento que fuera en la dirección de la auto-limitación. En sus palabras: "A la parálisis de nuestra sociedad moderna se le da el nombre de crisis de la energía. Para resolverla, es necesario alejar la ilusión que nuestra prosperidad depende del número de esclavos que nos abastecen de la energía que disponemos. Para esto es necesario determinar el umbral en el cual la energía corrompe, y unir a toda la comunidad en un proceso político que alcance este conocimiento y que funde sobre éste una auto-limitación" (Illich 2003 b: 390). Illich llama a este proceso una contra-investigación, ya que va en la dirección contraria a las investigaciones que se presentan en la comunidad científica en este dominio. 


\section{Illich como inspirador del decrecimiento}

La postura de Illich frente a la "crisis energética" no deja muchas líneas de acción posibles para orientar la solución de los problemas energéticos. "La dinámica del sistema industrial actual da el fundamento para su inestabilidad: está organizada en vista de un crecimiento indefinido, y la creación ilimitada de nuevas necesidades -las cuales se transforman rápidamente en limitaciones en el cuadro industrial [...] Un proceso tal de crecimiento hace al hombre una petición incongruente: encontrar la sumisión en la lógica de la herramienta. Luego, la estructura de la fuerza productiva da forma a las relaciones sociales" (Illich 2003a: 507).

Como ya hemos expuesto anteriormente, el crecimiento exacerbado encierra para Illich dos problemas. De una parte está la situación que podríamos llamar de finitud, es decir, la imposibilidad lógica de pretender un crecimiento infinito, por mucho que éste busque satisfacer este conjunto siempre creciente de necesidades. En segundo lugar, tenemos un problema de equidad. Un crecimiento indefinido de la producción de la energía conlleva serios problemas en la distribución y en el uso de esta energía, desfavoreciendo a los que tienen menos poder para justificar su "necesidad" de más energía.

Una serie de intelectuales ligados en algún momento con Illich, como Serge Latouche, Andre Gorz y otros, han sistematizado estas ideas en un término: el decrecimiento. Utilizando el paradigma de Illich, así como fuertemente las ideas de Georgescu-Roegen referentes a la aplicación de la idea de la entropía (Georgescu-Roegen, 2006) a la economía ${ }^{8}$, este grupo de pensadores aboga por abrir como única posibilidad viable de supervivencia para nuestra civilización la limitación del crecimiento económico, lo cual incluye la limitación con respecto a la producción de la energía.

Según Ridoux, el decrecimiento es "una disminución regular del consumo material y energético, en los países y para las poblaciones que consumen más que lo que es admisible por la impronta ecológica, evacuando prioritariamente el material superfluo, en beneficio del crecimiento de las relaciones humanas" (Ridoux 2006: 91-92). Esta disminución debe ser implementada considerando dos condiciones importantes: que ésta sea sustentable, es decir, que se realice de una manera progresiva y democrática; y por otra parte, que sea realizada en equidad. Estas dos serían condiciones necesarias (pero probablemente no suficientes) para lograr un proceso de decrecimiento en nuestra sociedad.

\footnotetext{
${ }^{8}$ En realidad se trata de "exportar" el concepto de entropía, desarrollado en la termodinámica, a la economía. Que esto sea válido, es de ya una pregunta importante a responder.
} 
Esta noción no tiene pocas críticas. ¿Cómo instaurar una cultura de la limitación, si existen seres humanos que ni siquiera pueden satisfacer sus necesidades básicas? Su implementación requeriría un cambio de conciencia, la liberación de lo que Serge Latouche

denomina las "mentes colonizadas" (Latouche 2006: 160). El proceso partiría con los países desarrollados, quienes son los que principalmente sobre-consumen. Pero eso no quiere decir que los países más pobres debieran dar rienda suelta a su consumo tampoco. La idea es transformar la manera de ver el fenómeno del sobre-consumo como una fuente de peligro para la supervivencia de nuestra civilización: "un crecimiento ilimitado no puede sino que transformarse en desmesura, en un delirio productivista, y conducir directamente al agravamiento de los problemas que constatamos hoy en día” (Ridoux 2006: 93).

Para lograr esto es necesario considerar una filosofía de vida basada en la "simplicidad voluntaria" (Ridoux 2006: 95). Se trata de una transformación de la manera de ver el mundo y de una implicación en éste. De ahí la importancia que otorga este movimiento al desarrollo de una democracia participativa y directa. En el lenguaje de Illich, se trata de construir una sociedad que sea convivencial y que, por lo tanto, esté consciente de su finitud y de la importancia de replantearse lo realmente necesario en sus necesidades.

\section{Conclusiones y Comentarios}

\section{Sobre la energía y el decrecimiento}

La postura del decrecimiento - como hemos visto basada fuertemente en la crítica illichiana- implica una postura individual que no es evidente. Un primer problema es el problema micro, de lo que ocurre en la psiquis individual frente a esta proposición. Porque detrás de esto está el problema de las necesidades y de cómo nos posicionamos frente a ellas. Gran parte del problema está relacionado con nuestra forma de plantearnos frente a lo que "necesitamos", en relación a los mecanismos de la psique humana. El poner esto en cuestión -el lograr esta "simplicidad voluntaria" de los "decrecientes"- no es algo evidente, pero nos indica un punto importante a considerar: la crisis de la energía refleja de cierta manera una crisis interior del ser humano y de la civilización.

Un segundo problema es el tema macro. Una postura decreciente implicaría, a nivel social, una serie de problemas. ¿Implicaría el decrecimiento aumentar las tasas de cesantía? Y si es así, ¿cómo haríamos frente a una recesión tal? Es por esto que una política en esta línea no puede ser concebida si no es con una transformación radical de nuestra forma de hacer las cosas. Un decrecimiento, por ejemplo, no podría ser impuesto por ley -a menos de encontrarnos en el escenario de una catástrofe natural, en cuyo caso sería la ley de la naturaleza la que lo impondría- sino que debe ser un movimiento político que traiga aparejado un cambio en la forma de ver las cosas. La sociedad convivencial de Illich está 
fundada en un individuo que es conciente de la importancia de esta actitud para su propio desarrollo y para el desarrollo de su comunidad.

El decrecimiento tiene en común con las ideas de Illich lo auto-evidente. ¿Cómo pensar un crecimiento sostenible, en un mundo finito? La contradicción salta a la vista, a pesar de que políticamente no sea tal vez la idea más correcta. Un decrecimiento implica también un volver a dar a las pequeñas unidades productivas el poder de tomar decisiones, el hecho de realmente ejercer una democracia participativa. ¿Es esto interesante para quienes detentan el poder en las comunidades actuales o es más bien visto como una amenaza? En mi opinión, el problema es el cómo. ¿Cómo generar un mecanismo que permita tomar conciencia de esta finitud y cómo tomar las medidas políticas, económicas y técnicas que estén a la altura de esta conciencia? Obviamente todo esto trae aparejado una transformación de nuestra comprensión del mundo, una transformación interior. Nobleza obliga.

\section{Sobre la posibilidad de un humanismo radical}

La idea de un "humanismo radical" como lo propone Erich Fromm en relación a las ideas de Illich nos parece un elemento interesante a la hora de reflexionar sobre lo que significa realmente trabajar en el área de la crítica. Ya sea en el tema de la energía, de la educación o cualquier otro aspecto de la sociedad, la importancia de mantener una mirada libre y no ser condicionado por estructuras teóricas e ideológicas -muchas veces omnipresentes por nuestra formación, o por la influencia de nuestros medios ambientes ideológicos- es esencial. Desde este punto de vista, es necesario ir más allá los discursos críticos habituales y crear nuevos espacios, por mucho que éstos puedan parecer utópicos. Creemos que Ivan Illich contribuyó mostrándonos la importancia de esto.

Por otra parte, el culto a lo técnico, a lo empírico, choca con esta tendencia a la utopía creadora. Pero es necesario también analizar esto con cuidado. ¿Cuántas políticas se justifican en estudios "empíricos" y "científicos"? La invocación a la técnica como justificadora de lo real tiene muchas veces un carácter religioso. Tenemos fe en la técnica, pero muchas veces no hacemos el esfuerzo de comprender realmente lo que esconde un informe o un argumento de este tipo. Illich notaba no sin cierta congoja que "vivimos en una sociedad extraña, donde la gente cree que actúa en base a una experiencia empírica" (Caylley 1996: 105). Una postura humanista radical nos obliga a ver las cosas intentando limpiarnos de los presupuestos o de los marcos teóricos, poniendo al hombre en el centro y no a las ideologías.

¿Es esto posible? ¿Podemos realmente no estar condicionados por estos elementos a la hora de intentar ver la realidad y elaborar una crítica, que quiera transformarla? No lo sabemos. Pero sí sabemos que se trata de un intento, que además nos lleva a reencontrar la idea de base de una teoría crítica. En su ensayo de 1930, Horkheimer plantea una diferencia 
fundamental entre lo que él llama una «teoría tradicional» y una «teoría crítica»: "en la época actual sin embargo, no es en las ciencias de la naturaleza, fundadas en la matemática presentada como un logos eterno, que el hombre puede aprender a conocerse a si mismo, es en una teoría crítica de la sociedad, tal que ella esté inspirada por la preocupación de establecer un orden conforme a la razón" (Horkheimer 1974: 27). En este sentido, el esfuerzo de observar la realidad sin repetir argumentos "implantados" en nosotros mismos, requiere coraje y libertad interior. Esto nos pone en un camino de construcción y de liberación de sí, en la tarea de la transformación de la realidad. Nos transformamos encontrándonos en esa búsqueda de libertad de ideas y cuadros impuestos- al mismo tiempo que buscamos transformar nuestra realidad.

La reflexión en torno a la crisis energética, a lo que necesitamos y a la importancia de poner en cuestión nuestra actitud frente al consumo de esta energía, es un mensaje que pensamos debemos a Ivan Illich. En una entrevista realizada en 1999, en Cuernavaca, Illich comentaba: "necesito mi biblioteca, hay ahí trazos de mi vida. Verá, cuando me viene una idea nueva, necesito verificar si ella no ha sido ya enunciada por otros. La Internet no me es suficiente" (Djian, 1999). Las necesidades profundas de cada uno son en realidad un reflejo de nuestras vidas. 


\section{Bibliografía}

Assoun, Paul-Laurent (2001, p.e. 1990), L'École de Frankfort, Paris, PUF.

Berman, M. (1992), Cuerpo y Espíritu, Cuatro Vientos, Santiago.

Cayley, David (1996), Entretiens Avec Ivan Illich, Bellarmin, Quebec.

Delobelle, Bernard (2008), Décroissance ou Catastrophe, mémoire M2 en Sciences de l'éducation, Universidad de Paris 8, no publicada.

Djian, Jean-Michel (1999), «Illich, le visionnaire injustement oublié», en: Le Monde de l'Éducation, Julio-Agosto, 1999, Paris.

Esteva, Gustavo (2005), Interview with Gustavo Esteva :The Society of the Different, In Motion Magazine, disponible en : www.inmotionmagazine.com/global/gest_int_1.html

Gajardo, Marcela (1993), «Ivan Illich», en: Perspectives, revue trimestrielle d'éducation comparée. Vol XXIII, UNESCO, BIE. Paris.

Georgescu-Roegen (2006) La décroissance: entropie, écologie, économie. Ellébore-Sang de la terre, Paris.

Horkheimer, Max (1974), Théorie Traditionnelle et Théorie Critique, Gallimard, Paris.

Illich, Ivan (1990) «Needs·, en: (2004), La Perte du Sens, Fayard, Paris.

Idem (2003) CEuvres Complètes V.1., Fayard, Paris.

Idem (2003a, p.e. 1973), «La Convivialité», en: CEuvres Complètes V.1., Fayard, Paris.

Idem (2003b , p.e. 1975), «Energie et équité», en: CEuvres Complètes V.1., Fayard, Paris.

Idem (2004), La Perte du Sens, Fayard, Paris.

Jay, Martin (1977), L’imagination Dialectique: L'école de Francfort 1923-1950, Payot, Paris.

Kellner, Douglas (1975), «The Frankfort School Revisited: A Critic of Martin Jay's Dialectics of Imagination», en: New German Critique, \#4 (1975), Duke University Press, Durham. 
Latouche, Serge (2006), Le pari de la décroissance, Fayard, Paris.

Ridoux, Nicolas (2006), La décroissance pour tous, Parangon. Lyon.

Stuchul, D., Esteva, G., Prakash, M. (sin fecha), From a Pedagogy of Liberation to Liberation from Pedagogy, descargado el 10/02/2008 del sitio http://www.swaraj.org/shikshantar/gustavols3.htm

Vincent, Jean - Marie (1976), La Théorie Critique de L'école de Francfort, Galilée, Paris.

Wiggerhaus, Rolf (1993), L'École de Frankfort: Histoire, Développement, Signification, PUF, Paris. 